\title{
BMJ Open Rehabilitation interventions to improve patient-reported outcomes and physical fitness in survivors of muscle invasive bladder cancer: a systematic review protocol
}

\author{
Elke Rammant, ${ }^{1}$ Renée Bultijnck, ${ }^{1}$ Nora Sundahl, ${ }^{1}$ Piet Ost, ${ }^{1}$ Nele S Pauwels, ${ }^{2}$ \\ Benedicte Deforche, ${ }^{3}$ Ronny Pieters, ${ }^{4}$ Karel Decaestecker, ${ }^{4}$ Valérie Fonteyne ${ }^{1}$
}

To cite: Rammant $\mathrm{E}$,

Bultijnck R, Sundahl N, et al. Rehabilitation interventions to improve patient-reported outcomes and physical fitness in survivors of muscle invasive bladder cancer: a systematic review protocol. BMJ Open 2017;7:e016054. doi:10.1136/ bmjopen-2017-016054

- Prepublication history and additional material are available. To view these files please visit the journal online (http://dx.doi.org/ 10.1136/ bmjopen-2016-016054)

Received 20 January 2017 Revised 12 April 2017 Accepted 13 April 2017

\section{CrossMark}

${ }^{1}$ Department of Radiation Oncology and Experimental Cancer Research, Ghent University Hospital, Ghent, Belgium

${ }^{2}$ Knowledge Center Ghent, Ghent University Hospital, Ghent, Belgium

${ }^{3}$ Department of Public Health, Ghent University, Ghent, Belgium ${ }^{4}$ Department of Urology, Ghent University Hospital, Ghent, Belgium

Correspondence to Miss Elke Rammant; elke.rammant@ugent.be

\section{ABSTRACT}

Introduction Survivors of muscle invasive bladder cancer (MIBC) experience physical and psychosocial side effects of cancer diagnosis and treatment. These negative side effects have a crucial impact on their health-related quality of life (HRQoL). To date, there is evidence that rehabilitation interventions such as physical activity and psychosocial support have a positive effect on the HRQoL of cancer survivors. Unfortunately, there are no specific guidelines for rehabilitation or survivorship programmes for MIBC survivors. Therefore, this systematic review aims to assess the effects of exercise-based and psychosocial rehabilitation interventions in MIBC survivors.

Methods and analysis The approach of this review is consistent with the Cochrane methodology. Randomized controlled trials and non-randomised studies will be included. The population of interest is patients ( $\geq 18$ years of age) with diagnosis of MIBC or high-risk non-MIBC for whom a radical cystectomy is indicated. There will be two eligible intervention types for inclusion: exercise-based and psychosocial rehabilitation interventions. The primary outcome measures are patient-reported outcomes (eg, $\mathrm{HRQ}$ oL, fatigue and pain) and physical fitness. Studies will be identified independently by two review authors by searching the Cochrane Central Register of Controlled Trials, MEDLINE, Embase, Web of Science and the Physiotherapy Evidence Database. A third reviewer will be asked by disagreements. Risk of bias will be assessed using the Cochrane Collaboration tool and the NewcastleOttawa Scale. Data will be summarised descriptively. If homogeneity of the studies is sufficient, meta-analysis will be undertaken. The broad scope of this review (ie, different interventions and study designs) is needed to have a comprehensive view on effective rehabilitation interventions.

Ethics and dissemination Ethics approval is not required, as no primary data will be collected. Results will be disseminated through a peer-reviewed publication.

\section{BACKGROUND}

\section{Description of the condition}

Bladder cancer (BC) is the 11th most common cancer worldwide. When both
Strengths and limitations of this study

- The overall approach of this review is consistent with the methodology described in the Cochrane Handbook for Systematic Reviews of Interventions.

- It is an innovative topic in the field of bladder cancer.

- Actual problem because the incidence of bladder cancer is rising.

- Possible paucity of studies meeting the inclusion criteria.

genders are considered separately, BC in men is rising to the seventh place, while $\mathrm{BC}$ in women is dropping to the 17 th place of most common cancer worldwide. ${ }^{12}$ Since BC is mainly diagnosed at more advanced age, the incidence is expected to raise due to an increased life expectancy. ${ }^{34}$ Thirty per cent of the BC patients are diagnosed with muscle invasive BC (MIBC, stages T2-T4) ${ }^{5}$ and up to $45 \%$ of patients with non-MIBC (NMIBC) will eventually progress to MIBC. ${ }^{6}$ The standard treatment of MIBC is neo-adjuvant chemotherapy followed by radical cystectomy combined with an extended pelvic lymph node dissection and urinary diversion (continent or incontinent bladder replacement). Also patients with high-risk NMIBC can be offered a radical cystectomy. ${ }^{1}$ This aggressive approach is associated with a variety of negative side effects, mainly hampering urinary, gastrointestinal and sexual function. This can lead to a loss of health-related quality of life (HRQoL), which refers to the patients' own perceptions of their health and ability to function ${ }^{7}$ encompassing physical, psychological, social and spiritual dimensions. ${ }^{8}$ Bladder-preserving radiochemotherapy is an alternative for radical cystectomy. Both radiotherapy and chemotherapy can also be used in the 
adjuvant or palliative setting and can cause important treatment-related side effects. Therefore, follow-up care beyond the acute diagnosis and treatment phase is necessary. ${ }^{9}$

\section{Description of the intervention}

The World Health Organization (WHO) has defined rehabilitation as 'the use of all means aimed at reducing the impact of disabling and handicapping conditions at enabling people with disabilities to achieve optimal social integration'. ${ }^{10} \mathrm{~A}$ more specific definition of rehabilitation in the setting of cancer is the following: "cancer rehabilitation is a concept that is defined by the patient and involves helping a person with cancer to obtain maximum physical, social, psychological, and vocational functioning within the limit by the disease and its treatment'. ${ }^{11}$ Therefore, cancer rehabilitation needs to comprise different intervention approaches. ${ }^{12} 13$

Physical activity (PA) is seen as one of the rehabilitation interventions to improve patient's quality of life (QOL) and survival outcomes. PA is defined as 'any movement created by the skeletal muscles that causes a substantial increase in energy expenditure'. ${ }^{14}$ It is important to point out that 'physical activity' and 'exercise' are not similar. PA can be seen as an overarching term that includes exercise as well as other activities that involve bodily movement and are done as part of playing, working, active transportation, house chores and recreational activities. Exercise, however, is a subcategory of PA. It is planned, structured, repetitive and purposeful to improve or maintain one or more components of physical fitness (cardiorespiratory fitness, muscular strength, muscular endurance, flexibility and body composition). ${ }^{15}$ These components represent important outcomes in cancer survivors that may mediate the influence of exercise on other outcomes. ${ }^{14}$

Psychosocial interventions are another important focus in rehabilitation. These sort of interventions aim to help patients cope with negative side effects of cancer diagnosis and treatment. ${ }^{16}$ Based on a framework proposed by Buffart et al, there are five categories of interventions: patient education, social support, coping skills training, psychotherapy and spiritual/existential therapy. ${ }^{17}$

\section{How the intervention might work}

In order to explain how cancer rehabilitation interventions might work, the revised Wilson and Cleary Model for HRQoL will be used as a conceptual framework. ${ }^{18}$ This is useful to explain the pathways between different patient outcomes. ${ }^{19}$ The model proposes five types of patient outcome measurements (biological function, symptoms, functional status, health perception and overall QOL), which have a causal relationship. ${ }^{1820}$ These five patient outcome measurements can be influenced by individual and environment characteristics.

In case of BC, morbidity associated with the disease and its treatment can lead to complications related to urinary diversion, urinary incontinence or constipation, sexual dysfunction, ${ }^{21}$ fatigue and psychological distress. ${ }^{22}$ This can potentially lead to a loss of physical, social, psychological and role function, which affects activities in daily life. As a consequence, the general health perceptions of the patient can be damaged, which can finally lead to an overall impaired QOL. All of these outcomes can be influenced by environment and individual characteristics.

Offering cancer rehabilitation can be seen as a physical environment characteristic. Since it has been proven in other cancer populations that cancer rehabilitation interventions have a positive influence on, for example, physical fitness, ${ }^{23}$ muscle capacity, ${ }^{24}$ fatigue and emotional distress, ${ }^{25}$ we can conclude, according to the revised Wilson and Cleary Model for HRQoL, that cancer rehabilitation interventions can have a positive influence on other patient outcomes such as HRQoL. ${ }^{18}$

Individual characteristics, such as advanced age and the associated increased risk of comorbidities, are important factors to take into account in patients with BC. These characteristics are associated with poorer health ${ }^{26}$ such as functional and psychosocial declines. ${ }^{27}$ Although this supports the need for cancer rehabilitation, the older age of patients with $\mathrm{BC}$ creates challenges in recommending rehabilitation interventions. Potential difficulties are the lack of social support in older patients and the need for extra time and resources to enrol these patients. ${ }^{28}$ Additionally, the high prevalence of urinary complications and problems with body image in patients with $\mathrm{BC}$ can act as potential barriers to participate in exercise interventions. ${ }^{26}$ According to Karvinen et al, exercise interventions for BC survivors should focus on offering enjoyable activities, education on the benefits of regular exercise, improving activity levels in important others and targeting perceived barriers. They also note that adjuvant therapy, age and invasiveness of the tumour may affect exercise participation. ${ }^{26}$ Furthermore, BC survivors seem to be most interested in walking and home-based, individual exercises that are not supervised. ${ }^{29}$

\section{Why it is important to do this review}

With an increasing number of cancer survivors, cancer rehabilitation will become imperative in cancer survivorship. In order to develop evidence-based rehabilitation programmes for patients with $\mathrm{BC}$ who received a curative treatment, it is essential to have a global picture of effective rehabilitation interventions. Therefore, a systematic review assessing the effects of exercise-based and psychosocial rehabilitation interventions inpatients with BC is needed. A previous systematic review assessed the associations of lifestyle factors (diet, smoking and PA) on HRQoL in BC survivors. Findings of this review concluded that there was limited evidence to support a positive association between HRQoL and PA in BC survivors. ${ }^{30}$ Our review differs with previous review in several aspects. First, we want to identify well-defined interventions that are effective. In previous review, they evaluated the PA pattern of the patient, which is not the same as an exercise intervention. Furthermore, this 
review will assess multiple other outcomes in addition to HRQoL (explained below). Additionally, to our knowledge, there is no systematic review that has summarised the evidence of psychosocial rehabilitation interventions in MIBC survivors in a systematic manner.

So far, no specific guidelines exist for exercise-based and/or psychosocial rehabilitation interventions for MIBC survivors. This review could give guidance to the development of specific evidence-based guidelines. Although this review focuses only on the exercise and psychosocial part of rehabilitation, it is important to note that rehabilitation of patients with cancer requires also other interventions such as diet counselling, smoking cessation and so on. ${ }^{12}$

It should also be noticed that providing cancer rehabilitation is an often-neglected facet of cancer care in terms of health policy and infrastructure. ${ }^{31}$ Frequently reported barriers to rehabilitation interventions are the lack of expertise, inappropriate referrals by physicians, funding issues $^{24}{ }^{31}$ and availability of rehabilitation resources. ${ }^{32}$ The results of this review may increase the awareness of physicians and funders of the importance of cancer rehabilitation.

\section{AIMS AND OBJECTIVES Primary objective}

Assessing the effects of rehabilitation interventions (exercise-based and psychosocial interventions) on patient-reported outcomes (PROs) (eg, QOL, fatigue and pain) and physical fitness in MIBC survivors.

\section{Secondary objective}

Identifying significant moderators of the intervention effects.

\section{METHODS}

The overall approach of this review is consistent with the methodology described in the Cochrane Handbook for Systematic Reviews of Interventions ${ }^{33}$ and is described below. Reports of current systematic review protocol adhere to the Preferred Items for Systematic Reviews and Meta-Analysis Protocol (PRISMA-P) checklist. ${ }^{34}$ The systematic review itself will adhere to the PRISMA. ${ }^{35}$

\section{Criteria for considering studies for this review \\ Types of studies}

Because of the anticipated low amount of randomised controlled trials (RCTs), RCTs and non-randomised studies (NRS) (ie, cohort studies, case-control studies, cross-sectional studies and quasi-randomised controlled clinical trials) will be considered as appropriate study designs. Results from RCTs and NRS will be presented separately.

\section{Types of participants}

The population of interest will be adults ( $\geq 18$ years of age) with medically confirmed diagnosis of localised
MIBC or high-risk NMIBC for whom a radical cystectomy is indicated. Studies with a majority of patients with metastasised BC will be excluded. Demographic factors are no exclusion criteria except for age ( $<18$ years of age). Studies involving participants with a range of cancers or other diagnoses that report results specifically for patients with MIBC will be included. Studies involving participants with urological cancers where data are not provided separately for patients with MIBC will be excluded.

\section{Types of interventions}

Exercise-based rehabilitation interventions considered for this review will be aerobic or endurance activities, strength or resistance training, balance exercises, flexibility exercises (with inclusion of yoga and Pilates), exercises specific to address sexual functioning and pelvic floor exercises in case of bladder preserving strategies or bladder reconstruction after radical cystectomy. Generalised advice to engage in regular PA activity will not be considered as an exercise-based rehabilitation intervention. In this review, the focus will be placed on exercise in a planned, structured, repetitive and purposeful rehabilitation intervention. PA as part of playing, working, active transportation, house chores or recreational activities will not be included.

Psychosocial rehabilitation interventions eligible for inclusion will be based on the framework proposed by Buffart et al: patient education (eg, stoma management, generalised advice to engage in PA activity), social support, coping skills training, psychotherapy and spiritual/existential therapy. ${ }^{17}$ Complementary medicine and therapies will not be included in this review.

Both intervention types can either be individual or in group, hospital based or home based (with follow-up by a professional), supervised by a physiotherapist or not and can be given before, during and/or after treatment. No limits will be placed on the timing, frequency, intensity and duration of rehabilitation interventions. The interventions will be compared with an inactive control intervention (eg, no treatment, standard care or a waiting list control).

\section{Types of outcome measures}

For both primary and secondary outcome measures, there will be no exclusion based on length of follow-up.

\section{Primary outcomes}

1. PROs including overall HRQoL, specific HRQoL domains including symptoms such as fatigue, pain, urinary incontinence, sexual dysfunction, gastrointestinal dysfunction and psychological factors such as anxiety, depression, stress and selfesteem. Due to the wide range of questionnaires used for PROs and the non-consensus of using one standardised questionnaire, only studies using the standardised and validated measurement instruments for PRO, found in Table $1,{ }^{36-48}$ will be included. All studies using other measurement 
Table 1 Standardised and validated measurement instruments for the included patient-reported outcomes

\begin{tabular}{|c|c|}
\hline Patient-reported outcome & Validated measurement instrument \\
\hline HRQoL & $\begin{array}{l}\text { EQ-5D visual analogue scale, SF-36, Ferrans and Powers QLI, WHO QOL, SF-12, Padilla } \\
\text { QLI, SF-20 and Satisfaction With Life Scale }\end{array}$ \\
\hline Bladder cancer-specific QOL & EORTC QLQ-BLM30, BCI and FACT-BI \\
\hline Sexual function & IIEF-5 and FSFI \\
\hline \multicolumn{2}{|l|}{ Psychological factors } \\
\hline $\begin{array}{l}\text { Depression } \\
\text { Anxiety } \\
\text { Stress } \\
\text { Self-esteem }\end{array}$ & $\begin{array}{l}\text { BSI-18, BDI,ISR-depression scale } \\
\text { STAI anxiety scales, numeric rating scales or visual analogue scale } \\
\text { Perceived Stress Scale } \\
\text { Rosenberg self-esteem scale }\end{array}$ \\
\hline
\end{tabular}

instruments will be excluded, unless proof of their validation can be found in literature.

2. Physical fitness assessed by $\mathrm{VO}_{2}$ peak, $\mathrm{VO}_{2}$ max, 6 or $12 \mathrm{~min}$ walk test, $400 \mathrm{~m}$ walk test, handgrip strength tests, sit and reach tests or other proven to be validated instruments.

\section{Secondary outcomes}

1. Cancer recurrence, cancer-specific survival, progression-free survival, overall survival, mortality, years of life lost or 5-year survival.

2. Body composition assessed by height, weight, body mass index, muscle capacity, fat mass, lean body mass, thickness of skin folds, body fat, arm circumference, waist circumference, hip circumference or waist-hip ratio.

3. Bone mineral density or fracture risk assessed by fracture risk assessment tool. ${ }^{49}$

4. Karnofsky performance score. ${ }^{50}$

\section{Search methods for identification of studies \\ Electronic searches}

The following electronic databases will be searched from inception until the search date: the Cochrane Central Register of Controlled Trials (CENTRAL), MEDLINE (using the PubMed interface), Embase (using the embase. com interface), Web of Science and the Physiotherapy Evidence Database (PEDro). The search strategies will be evaluated using the Peer Review of Electronic Search
Strategies (PRESS) checklist ${ }^{51}$ and will be monitored and peer reviewed by an information specialist (NSP). The search strategies are presented in online supplementary file 1 .

\section{Searching other resources}

The cited and citing references of the included studies will be checked via Web of Science.

\section{Data collection and analysis}

\section{Selection of studies}

All references found through the search process will be downloaded in a database created by reference management software (Endnote). After removing duplicates in Endnote, all references will be imported into Covidence for screening purposes. Obviously irrelevant studies, based on title and abstract, will independently be excluded by two review authors (ER and VF). After screening the titles and abstracts, two review authors (ER and VF) will independently assess full-text reports for eligibility. Discrepancies will be discussed with a third review author (NS). Reasons for exclusion of full-texts will be documented. Studies will be excluded if no full-text is available. Abstracts in any other language than English will be excluded. There will be no language restriction for full texts, and translations will be carried out if necessary. If studies have multiple publications with the same outcome(s) reported, manuscripts with the longest follow-up will be selected for inclusion. Older publications 
referred to in included articles will be accessed to clarify methods if required.

\section{Data extraction}

A modified Effective Practice and Organisation of Care (EPOC) data collection form of the Cochrane collaboration will be used and pilot tested with at least three studies in the review. ${ }^{52}$ After pilot testing the form, adjustments can be made. Data extraction will be performed independently by two review authors (ER and VF). For each included study in the review, at least following information will be extracted:

- General information: date form completed, name of person extracting data, report title, report ID, authors' names, source, country, contact address, language of publication and year of publication.

- Population and setting: population description (from which study participants are drawn), setting (eg, inpatient, outpatient, hospital setting, home setting and combination) and inclusion and exclusion criteria.

- Methods: aim of study, design, unit of allocation, start and end date and duration of participation.

- Participants: number of participants in intervention and control groups, details of clusters if applicable, baseline imbalances, participant demographics such as sex and age, disease-related characteristics such as stage of disease, received treatment(s) and comorbidities.

- Intervention: type of intervention (exercise based, psychosocial or combination), details of intervention type (eg, aerobic, pelvic floor exercises, counselling, patient education), cointervention(s), type of control intervention, frequency, duration and providers of the intervention.

- Outcomes: outcome name, time points measured and reported, outcome definition, person measuring/reporting, upper and lower limits of scales, unit measurement if relevant, if outcome/ tool is validated, imputation of missing data and assumed risk estimate if reported.

- Results: outcome, measurement effects (please see data analyses below) for intervention and comparison group, baseline data, number of missing participants and reasons and statistical methods used.

\section{Assessment of risk of bias in included studies}

The Cochrane Collaboration's tool for assessing risk of bias will be used for RCTs. Assessment of risk of bias in NRS will be done using the Newcastle-Ottawa Scale for observational studies. The assessment of risk of bias will be done independently by two reviewers (ER and NSP). Differences will be discussed and a third reviewer (NS) will be consulted when needed. Results will be summarised both in a graph and a narrative summary. In order to evaluate selective reporting, the reviewers will check clinical trial registries or search any protocols of the studies for a priori reported primary and secondary outcome measures. The strength of the body of evidence will be assessed according to the GRADE approach.

\section{Dealing with missing data}

If essential data are not available in the publication, we will first attempt to contact the study authors. If this is not possible, we will try to back-calculate from the data presented. If data will be obtained from other study authors, this will be reported in the review in a transparent manner. This way, we can keep in mind that these missing data obtained from study authors were not peer reviewed. Studies assessing lifestyle interventions may have issues with compliance. Therefore, reasons for missing data (eg, dropouts, losses to follow-up and withdrawals) will be carefully reported.

\section{Assessment of heterogeneity}

First, there will be a critical consideration of the heterogeneity between the different interventions and outcomes to evaluate whether there is clinical diversity. Based on this evaluation, there will be a decision if a meta-analysis can be conducted. When there's no clinical heterogeneity, statistical heterogeneity will be quantified using the $I^{2}$ statistic. We will consider the statistical heterogeneity to be high if $I^{2}>50 \%$. Depending on the heterogeneity of the studies and their results, we will further decide if a meta-analysis can be conducted. We will attempt to explain any observed heterogeneity in the review.

\section{Assessment of reporting bias}

Funnel plots will be used to assess publication bias when 10 or more studies are included in a meta-analysis.

\section{Data synthesis}

The findings from the included studies will be summarised descriptively. For dichotomous outcomes, measurement of treatment effect will be reported as risk ratios and 95\% CIs. For continuous outcomes, we will calculate mean differences and $95 \%$ CIs when results are reported on the same scale (or can be converted to the same scale) or standardised mean differences and 95\% CIs if results are reported on different scales.

There will be an attempt to identify significant moderators, based on the most important demographical and clinical characteristics in this population. In order to do this, there will be a subgroup analysis for age and urinary diversion type. To prevent cointervention bias, there will also be a subgroup analysis based on the type of intervention (only exercised-based interventions, only psychosocial interventions or a combination).

When homogeneity of the studies is sufficient, random-effects meta-analysis will be undertaken separately for each type of study design. Only studies with low risk of bias will be included in meta-analyses. Therefore, we will perform sensitivity analyses to investigate how conclusions might be affected if studies at high or unclear risk of bias were included. 


\section{Ethics and dissemination}

Ethics approval is not required, as no primary data will be collected. Results will be disseminated through a peer-reviewed publication.

The scope of this review is broad (ie, different rehabilitation interventions and study designs). Although this means that heterogeneity might be present between the studies, evaluation of different intervention approaches is needed to develop optimal rehabilitation or survivorship programmes. Caution will be present in the interpretation of the results because of the fact that evidence from RCTs is higher than evidence from NRS. Therefore, the results derived from RCTs will be seen as the primary evidence. Results from NRS will be seen as additional evidence to support the results from RCTs.

This systematic review has several strengths. First, the overall approach of this review is consistent with the methodology described in the Cochrane Handbook for Systematic Reviews of Interventions. Second, rehabilitation interventions are an innovative topic in the field of $\mathrm{BC}$ so this review ensures an absolute value. A third strength is the fact that MIBC is an actual problem because of the potentially rising incidence of MIBC due to the ageing population.

The results of this systematic review could also have potential limitations in terms of biased results due to the nature of exercise and psychosocial interventions. It is impossible for such interventions to blind participants and personnel. Therefore, 'blinding of participants and personnel' will not be taken into account in the risk of bias assessment because of the thought that this will not necessarily affect the study quality. However, attrition and adherence biases and selective reporting biases are other common concerns around high risk of bias that would affect the study quality. ${ }^{53}$ Therefore, it is important that the risk of bias assessment will be carried out very carefully. Another limitation of this review could be the possible paucity of studies meeting the inclusion criteria.

In spite of these anticipated limitations, it is important to conduct this review because of the expected implications for healthcare, research and survivorship. To date, there are no specific guidelines for exercise-based or psychosocial rehabilitation interventions for MIBC survivors. This systematic review is expected to provide guidance to the development of specific guidelines and evidence-based rehabilitation or survivorship programmes for MIBC survivors. Development of such programmes could have further implications for healthcare if they will be translated into daily clinical practice.

By identifying those interventions that have a positive effect on patient outcomes and which underlying factors ensure the success of such rehabilitation interventions, new interventions can be developed that can contribute to further research. The positive influence of PA on survivorship is already suggested in different tumour types. ${ }^{54}$ The results of this systematic review can contribute to patient survivorship from the hypothesis that this positive association is also applicable in MIBC.
Contributors ER drafted the manuscript and developed the methods and analyses strategy. VF coordinated the process. NSP and NS aided in developing the search strategy. VF, PO and KD provided uro-oncology-specific expertise. RB and BD gave valuable input concerning rehabilitation.

Funding This study is funded by a grant of Kom op tegen Kanker. The granting bodies are not involved in data collection or analysis.

Competing interests None declared.

Provenance and peer review Not commissioned; externally peer reviewed.

Data sharing statement There is no additional unpublished data.

Open Access This is an Open Access article distributed in accordance with the Creative Commons Attribution Non Commercial (CC BY-NC 4.0) license, which permits others to distribute, remix, adapt, build upon this work non-commercially, and license their derivative works on different terms, provided the original work is properly cited and the use is non-commercial. See: http://creativecommons.org/ licenses/by-nc/4.0/

(c) Article author(s) (or their employer(s) unless otherwise stated in the text of the article) 2017. All rights reserved. No commercial use is permitted unless otherwise expressly granted.

\section{REFERENCES}

1. Witjes JA, Compérat E, Cowan NC, et al. EAU guidelines 2016: muscle invasive and metastatic bladder Cancer. https://uroweborg/ guideline/bladder-cancer-muscle-invasive-and-metastatic/ (accessed on 12/10/2016).

2. Burger M, Catto JW, Dalbagni G, et al. Epidemiology and risk factors of urothelial bladder Cancer. Eur Urol 2013;63:234-41.

3. Malats N, Real FX. Epidemiology of bladder cancer. Hematol Oncol Clin North Am 2015;29:177-89.

4. Shahrokh FS, Milowsky M, Droller MJ. Bladder Cancer in the elderly. Urologic Oncology 2009;27:653-67.

5. Stenzl A, Witjes J, Compérat E, et al. Bladder Cancer Muscleinvasive and metastatic. UPDATE 2012.

6. van Rhijn BW, Burger M, Lotan Y, et al. Recurrence and progression of disease in non-muscle-invasive bladder Cancer: from epidemiology to treatment strategy. Eur Urol 2009;56:430-42.

7. Wright JL, Porter MP. Quality-of-life assessment in patients with bladder Cancer. Nat Clin Pract Urol 2007;4:147-54.

8. Mohamed NE, Gilbert F, Lee CT, et al. Pursuing Quality in the application of bladder Cancer quality of Life Research. Bladder Cancer 2016;2:139-49.

9. National Cancer Institute. Division of Cancer control \& population sciences. 2016 https://cancercontrolcancergov/ocs/statistics/ definitionshtml (accessed on 11/10/2016).

10. Hojan K. Contemporary rehabilitation at Cancer centres. Journal of Medical Science 2016;83:156-60.

11. Gudbergsson SB, Dahl AA, Loge JH, et al. What is covered by "cancer rehabilitation" in PubMed? A review of randomized controlled trials 1990-2011. J Rehabil Med 2015;47:97-106.

12. : :87-101. Weis J, Giesler JM. Rehabilitation for cancer patients. Psycho-Oncology 2014.

13. Harrison JD, Young JM, Price MA, et al. What are the unmet supportive care needs of people with Cancer? A systematic review. Support Care Cancer 2009;17:1117-28.

14. McGowan EL, Courneya KS. Exercise interventions in supportive oncology, 2011.

15. World Health Organisation. Global Strategy on Diet. Physical Activity and Health http://wwwwhoint/dietphysicalactivity/pa/en/ (accessed on 11/10/2016).

16. Parahoo K, McDonough S, McCaughan E, et al. Psychosocial interventions for men with prostate Cancer. The Cochrane Library 2013.

17. Buffart LM, Kalter J, Chinapaw MJ, et al. Predicting OptimaL cAncer Rehabllitation and supportive care (POLARIS): rationale and design for meta-analyses of individual patient data of randomized controlled trials that evaluate the effect of physical activity and psychosocial interventions on health-related quality of life in Cancer survivors. Syst Rev 2013;2:1.

18. Ferrans CE, Zerwic JJ, Wilbur JE, et al. Conceptual model of healthrelated quality of life. J Nurs Scholarsh 2005;37:336-42.

19. Saban KL, Penckofer SM, Androwich I, et al. Health-related quality of life of patients following selected types of lumbar spinal surgery: a pilot study. Health Qual Life Outcomes 2007;5:71.

20. Hacker E. Exercise and quality of life: strengthening the connections. Clin J Oncol Nurs 2009;13:31-9. 
21. Jensen BT, Jensen JB, Laustsen S, et al. Multidisciplinary rehabilitation can impact on health-related quality of life outcome in radical cystectomy: secondary reported outcome of a randomized controlled trial. J Multidiscip Healthc 2014;7:301-11.

22. Palapattu GS, Haisfield-Wolfe ME, Walker JM, et al. Assessment of perioperative psychological distress in patients undergoing radical cystectomy for bladder Cancer. J Urol 2004;172:1814-7.

23. Buffart LM, Kalter J, Sweegers MG, et al. Effects and moderators of exercise on quality of life and physical function in patients with Cancer: an individual patient data meta-analysis of 34 RCTs. Cancer Treat Rev 2017;52:91-104.

24. Alfano CM, Ganz PA, Rowland JH, et al. Cancer survivorship and Cancer rehabilitation: revitalizing the link. J Clin Oncol 2012;30:904-6.

25. Andersen BL. Biobehavioral outcomes following psychological interventions for Cancer patients. J Consult Clin Psychol 2002;70:590-610.

26. Karvinen $\mathrm{KH}$, Courneya KS, Plotnikoff RC, et al. A prospective study of the determinants of exercise in bladder Cancer survivors using the Theory of Planned Behavior. Support Care Cancer 2009;17:171-9.

27. Brown JC, Winters-Stone K, Lee A, et al. Cancer, physical activity, and exercise. Compr Physiol 2012;2:2775-809.

28. Grabowski JP, Pintus Dla, Ramesh HSJ, et al. The Challenges of Treating Cancer in Older Patients - How Effective Are Clinical Trials in Developing Unique Treatments? Eur Oncol Haematol 2008;04:94-6.

29. Karvinen KH, Courneya KS, Venner P, et al. Exercise programming and counseling preferences in bladder Cancer survivors: a population-based study. J Cancer Surviv 2007;1:27-34.

30. Gopalakrishna A, Longo TA, Fantony JJ, et al. Lifestyle factors and health-related quality of life in bladder Cancer survivors: a systematic review. J Cancer Surviv 2016;10:874-82.

31. Pearson EJM, Twigg VJ. A framework for rehabilitation for Cancer survivors. Eur J Cancer Care 2013;22:701-8.

32. Bultijnck R, Surcel C, Ploussard G, et al. Practice patterns compared with Evidence-based strategies for the management of androgen deprivation Therapy-Induced Side Effects in Prostate Cancer Patients: Results of a European Web-based Survey. European Urology Focus 2016;2:514-21.

33. Higgins JPT, ed. Cochrane Handbook for Systematic Reviews of Interventions Version 5.1.0 [updated March 2011: The Cochrane Collaboration, 2011. Available from. www.handbook.cochrane.org.

34. Shamseer L, Moher $D$, Clarke $M$, et al. Preferred reporting items for systematic review and meta-analysis protocols (PRISMA-P) 2015: elaboration and explanation. BMJ 2015;349:97647.

35. Moher D, Liberati A, Tetzlaff J, et al. Preferred reporting items for systematic reviews and meta-analyses: the PRISMA statement. BMJ 2009;339:b2535

36. Urologic Oncology: Seminars and Original Investigations. Measuring health-related quality of life in men with prostate Cancer: a systematic review of the most used questionnaires and their validity. Elsevier, 2015

37. Danna BJ, Metcalfe MJ, Wood EL, et al. Assessing Symptom Burden in Bladder Cancer: an overview of bladder Cancer specific HealthRelated Quality of Life Instruments. Bladder Cancer 2016;2:329-40.
38. Vodermaier A, Linden W, Siu C. Screening for emotional distress in Cancer patients: a systematic review of assessment instruments. Natl Cancer Inst 2009;101:1464-88.

39. Brandt WA, Loew T, von Heymann F, et al. How does the ICD-10 symptom rating (ISR) with four items assess depression compared to the BDI-II? A validation study. J Affect Disord 2015;173:143-5.

40. Hornblow AR, Kidson MA. The visual analogue scale for anxiety: a validation study. Aust N Z J Psychiatry 1976;10:339-41.

41. Araujo IB, Castelani A, Soler T, et al. Stress assessment in the elderly by the perceived stress Scale (PSS). International Psychogeriatrics 2015;27:S119-S19.

42. Li CC, Chen ML, Chang TC, et al. Social support buffers the effect of self-esteem on quality of life of early-stage cervical Cancer survivors in Taiwan. Eur J Oncol Nurs 2015;19:486-94.

43. Gilbert SM, Dunn RL, Hollenbeck BK, et al. Development and validation of the bladder Cancer index: a comprehensive, disease specific measure of health related quality of life in patients with localized bladder Cancer. J Urol 2010;183:1764-70.

44. Dargis L, Trudel G, Cadieux J, et al. Validation of the International Index of Erectile function (IIEF) and presentation of norms in older men. Sexologies 2013;22:e20-e26.

45. Baser RE, Li Y, Carter J. Psychometric validation of the female sexual function index (FSFI) in Cancer survivors. Cancer 2012;118:4606-18.

46. Eypasch E, Williams JI, Wood-Dauphinee S, et al. Gastrointestinal Quality of Life Index: development, validation and application of a new instrument. Br J Surg 1995;82:216-22.

47. Hajebrahimi S, Nourizadeh D, Hamedani R, et al. Validity and reliability of the International Consultation on Incontinence Questionnaire-Urinary Incontinence Short form and its correlation with urodynamic findings. Urol $J$ 2012;9:685.

48. Gray M. Psychometric evaluation of the international prostate symptom score. Urol Nurs 1998;18:175.

49. Watts NB, Lewiecki EM, Miller PD, et al. National Osteoporosis Foundation 2008 Clinician's Guide to Prevention and Treatment of Osteoporosis and the World Health Organization Fracture Risk Assessment Tool (FRAX): what they mean to the bone densitometrist and bone technologist. J Clin Densitom 2008;11:473-7.

50. Mor V, Laliberte L, Morris JN, et al. The Karnofsky Performance Status Scale. an examination of its reliability and validity in a research setting. Cancer 1984;53:2002-7.

51. McGowan J, Sampson M, Salzwedel DM, et al. PRESS Peer Review of Electronic search strategies: 2015 Guideline Statement. J Clin Epidemiol 2016;75:40-6.

52. Effective Practice and Organisation of Care (EPOC). EPOC Resources for review authors. Oslo: norwegian knowledge Centre for the Health Services; 2015. secondary effective practice and Organisation of Care (EPOC). EPOC Resources for review authors. Oslo: norwegian knowledge Centre for the Health Services;. 2015 http://epoc.cochrane.org/epoc-specific-resources-review-authors.

53. Bourke L, Smith D, Steed L, et al. Exercise for men with prostate Cancer: a systematic review and Meta-analysis. Eur Urol 2016;69:693-703.

54. Ammitzbøll G, Søgaard K, Karlsen RV, et al. Physical activity and survival in breast Cancer. Eur J Cancer 2016;66:67-74. 